\title{
Structural and protein interaction effects of hypertrophic and dilated cardiomyopathic mutations in alpha-tropomyosin
}

\author{
Audrey N. Chang ${ }^{1 t}$, Norma J. Greenfield ${ }^{2}$, Abhishek Singh ${ }^{2,3}$, James D. Potter $^{1}$ and Jose R. Pinto ${ }^{*}$ \\ ${ }^{1}$ Department of Molecular and Cellular Pharmacology, Leonard Miller School of Medicine, University of Miami, Miami, FL, USA \\ 2 Department of Neuroscience and Cell Biology, Robert Wood Johnson Medical School, Rutgers University, New Jersey, NJ, USA \\ ${ }^{3}$ Department of Cardiology, UCSF Medical Center, University of California, San Francisco, San Francisco, CA, USA \\ ${ }^{4}$ Department of Biomedical Sciences, Florida State University College of Medicine, Tallahassee, FL, USA
}

Edited by:

Kenneth S. Campbell, University of Kentucky, USA

\section{Reviewed by:}

Seth L. Robia, Loyola University Chicago, USA

Ranganath Mamidi, Case Western

Reserve University, USA

\section{*Correspondence:}

Jose R. Pinto, Department of

Biomedical Sciences, Florida State

University College of Medicine, 1115

West Call Street, 1350-H,

Tallahassee, FL 32306-4300, USA

e-mail: jose.pinto@med.fsu.edu

${ }^{\dagger}$ Present address:

Audrey N. Chang, Department of

Physiology, University of Texas

Southwestern Medical Center,

Dallas, USA
The potential alterations to structure and associations with thin filament proteins caused by the dilated cardiomyopathy (DCM) associated tropomyosin (Tm) mutants E40K and E54K, and the hypertrophic cardiomyopathy (HCM) associated Tm mutants E62O and L185R, were investigated. In order to ascertain what the cause of the known functional effects may be, structural and protein-protein interaction studies were conducted utilizing actomyosin ATPase activity measurements and spectroscopy. In actomyosin ATPase measurements, both HCM mutants and the DCM mutant E54K caused increases in $\mathrm{Ca}^{2+}$-induced maximal ATPase activities, while E40K caused a decrease. Investigation of Tm's ability to inhibit actomyosin ATPase in the absence of troponin showed that HCM-associated mutant Tms did not inhibit as well as wildtype, whereas the DCM associated mutant E40K inhibited better. E54K did not inhibit the actomyosin ATPase activity at any concentration of Tm tested. Thermal denaturation studies by circular dichroism and molecular modeling of the mutations in Tm showed that in general, the DCM mutants caused localized destabilization of the Tm dimers, while the HCM mutants resulted in increased stability. These findings demonstrate that the structural alterations in Tm observed here may affect the regulatory function of $\mathrm{Tm}$ on actin, thereby directly altering the ATPase rates of myosin.

Keywords: tropomyosin structure, cardiomyopathy, circular dichroism, thermal denaturation, actomyosin ATPase, molecular modeling

\section{INTRODUCTION}

Numerous mutations in the proteins of the sarcomere have been associated with cardiomyopathies (Chang and Potter, 2005; Force et al., 2010; Seidman and Seidman, 2011; Hershberger et al., 2013). Various studies of cardiomyopathic mutations suggest distinct mechanisms for the progression of the dilated cardiomyopathic (DCM) and hypertrophic cardiomyopathic (HCM) phenotypes (Chang et al., 2005; Robinson et al., 2007; Willott et al., 2010). An understanding of the disease progression necessitates the study of the effects of the mutations on protein structure and function (Force et al., 2010; Tardiff, 2011). One of the sarcomeric proteins found to have mutations associated with cardiomyopathies is cardiac $\alpha$-tropomyosin ( $\mathrm{Tm}$ ) (Redwood and Robinson, 2013). Tm is a semi-flexible rod-like protein whose coiled-coil structure functions to control myosin interaction sites on actin (Perry, 2001). Troponin (Tn) confers $\mathrm{Ca}^{2+}$ sensitivity to this function (Holroyde et al., 1980; Farah and Reinach, 1995; Gordon et al., 2000). Putative actin on/off regions along the

Abbreviations: wild-type human $\alpha$-striated tropomyosin with and $\mathrm{N}$-terminal Ala-Ser dipeptide replacing the acetyl group (ASWT); DCM, dilated cardiomyopathy; HCM, hypertrophic cardiomyopathy; Tm, tropomyosin; $\mathrm{Tn}$, troponin. length of Tm have been known for some time (McLachlan and Stewart, 1976), and more recent studies of these regions have demonstrated that although the length of the Tm dimer is composed of regular turns which correspond to seven actin binding regions, those regions are not all equal in terms of structure and contribution to thin filament function (Phillips et al., 1986; Hammell and Hitchcock Degregori, 1997; Landis et al., 1997, 1999; Singh and Hitchcock-Degregori, 2003; Barua et al., 2013).

How a single point mutation out of 284 amino acids in Tm can lead to cardiomyopathies is an interesting question which this paper attempts to understand, through the study of four mutations, the DCM associated mutations $\mathrm{E} 40 \mathrm{~K}$ and $\mathrm{E} 54 \mathrm{~K}$, and the HCM associated mutations E62Q and L185R. All of these mutations were previously identified from patients who presented severe cardiomyopathy (Olson et al., 2001; Van Driest et al., 2002; Jongbloed et al., 2003). Previous investigation on the effects of these HCM and DCM mutations by myofibrillar ATPase measurements showed an increase in $\mathrm{Ca}^{2+}$ sensitivity for HCM and a decrease in $\mathrm{Ca}^{2+}$ sensitivity for DCM associated mutations, which is commonly found in mutations of the thin filament associated with these diseases (Chang et al., 2005). In addition, a decrease in inhibition of ATPase activity at low $\mathrm{Ca}^{2+}$ 
concentrations were reported for the HCM associated mutations; while DCM associated mutations had no significant effect on inhibition, suggesting that distinct mechanisms, at the thin filament level, may underlie the different disease phenotypes (Chang et al., 2005). The causes of the distinct functional effects previously reported are unknown, and while semi- "intact" systems such as reconstituted myofibrils provide important mechanistic information, there are too many variables to identify the causative perturbation.

Reconstituted fiber studies have shown distinct changes in actin binding properties and actomyosin interactions linked to altered movement of Tm on actin caused by DCM associated mutations E40K and E54K (Borovikov et al., 2009b,c; Bai et al., 2012). Results of such studies have led to conclusions that distinct mutations cause defects in contraction via mechanisms unique to the mutations (Borovikov et al., 2009a,b,c; Bai et al., 2012). Reconstituted fibers are complex intact systems in which the components of the thin filament are completely replaced with exogenous proteins. More recently, energy landscape studies have shown that mutations in Tm linked to HCM are associated with a decrease in actin-Tm interaction energy, which is thought to impair the relaxation properties of the thin filament (Orzechowski et al., 2014). However, using the same technique, mutations in Tm linked to DCM do not seem to enhance actin-Tm interaction (Orzechowski et al., 2014). Due to the complexity of the system, here we aimed to understand the effects of the mutations on Tm's interactions with its functional partner proteins by actomyosin ATPase activity assays and on the helical stability of Tm dimers. Unlike reconstituted myofibrillar or fiber preparations, actomyosin ATPase activity assays were performed with purified actin, myosin, $\mathrm{Tm}$ and Tn. By titrating $\mathrm{Tm}$ and $\mathrm{Tn}$ into actin and myosin mixtures, we sought to identify the specific protein-protein interaction affected by the abovementioned point mutations in Tm. Results of these studies pointed toward the possibility of effects of the mutations on Tm dimer interactions, which have the potential for long-range effects on myofilament function. The structural effects of the mutations were further dissected through thermal denaturation studies and energy minimization studies.

\section{MATERIALS AND METHODS EXPRESSION AND PURIFICATION OF RECOMBINANT TMS AND TNS}

Recombinant Tm and Tns were expressed and purified utilizing previously described methods (Chang et al., 2005). AlanineSerine were added to the N-terminus of all Tm proteins to mimic acetylation as previously described (Urbancikova and HitchcockDegregori, 1994), and is referred to as "ASWT" in figures. Human cardiac Tn complexes comprised of recombinant TnT, TnI, and TnC were prepared by sequential dialysis in decreasing concentrations of urea and $\mathrm{KCl}$ (Gomes et al., 2002).

\section{THERMAL DENATURATION STUDIES BY CIRCULAR DICHROISM (CD)}

Far-UV CD spectra were collected using a 1-mm-path quartz cell in a Jasco J-720 spectropolarimeter. The measurements were obtained using pure recombinant proteins which were dialyzed in $10 \mathrm{mM}$ sodium phosphate buffer $\mathrm{pH} 7.0,150 \mathrm{mM}$ sodium fluoride, and diluted to $0.1 \mathrm{mg} / \mathrm{mL}$. Thermal denaturation measurements were obtained with proteins which had been diluted to $0.1 \mathrm{mg} / \mathrm{mL}$, at $222 \mathrm{~nm}$ over a range of temperatures $\left(20-60^{\circ} \mathrm{C}\right)$. Melting temperatures were determined by fitting the mean residue ellipticity, $[\theta]_{222}$, to a two-state or threestate transition model as previously described (Greenfield and Hitchcock-Degregori, 1995; Greenfield, 2006). See description for the three-state transition model below:

$$
\theta_{\text {observed }}=\epsilon_{1} \alpha_{1}+\epsilon_{2} \alpha_{2}+\epsilon_{2} \alpha_{2}
$$

where

$$
\begin{aligned}
\alpha_{1} & =\frac{K_{1}}{1+K_{1}}, \alpha_{2}=\frac{K_{2}}{1+K_{2}}, \text { and } \\
\alpha_{3} & =\frac{\left(4[C] K_{3}+1-\sqrt{8[C] K_{3}+1}\right)}{4[C] K_{3}}
\end{aligned}
$$

and

$$
\begin{aligned}
& K_{1}=e^{\left(\frac{\Delta H_{1}}{R T}\right)\left(\frac{T}{T_{M 1}}-1\right)}, K_{2}=e^{\left(\frac{\Delta H_{2}}{R T}\right)\left(\frac{T}{T_{M 2}}-1\right)}, \\
& K_{3}=e^{\left(\frac{\Delta H_{3}}{R T}\right)\left(\frac{T}{T_{M 3}}-1\right)-\operatorname{In}[C]}
\end{aligned}
$$

$\Delta H_{1}, \Delta H_{2}$, and $\Delta H_{3}$ are the respective enthalpies of folding of the first, second, and third transitions at the lowest to highest temperature. $T_{M 1}, T_{M 2}$, and $T_{M 3}$ are the observed midpoints of each transition. $\in_{1}, \in_{2}, \in_{3}$ are the extinction coefficients for the circular dichroism change associated with each transition, and [C] is the concentration of the Tm dimer in mole/L. The initial values of $\Delta \mathrm{H}$ and $T_{M}$ were estimated and the unfolding equations were fit using SigmaPlot 11.0 (Greenfield, 2006). The ASWT and L185R were fit using a two-state transition model, while the other mutants were fit using a three-state transition model as determined by the correlation coefficient (Greenfield, 2006).

\section{PURIFICATION OF RABBIT SKELETAL ACTIN}

Skeletal actin was purified from the longissimus dorsi and psoas muscles of female New Zealand White rabbits, utilizing routine methods established in the lab, according to the procedure described by Strzelecka-Golaszewska et al. (1975) and Pardee and Spudich (1982).

\section{PURIFICATION OF PORCINE CARDIAC MYOSIN}

Cardiac myosin was purified from the left ventricles of hearts from freshly sacrificed pigs, by a method routinely utilized in the lab (Szczesna et al., 2000), based on the original procedure described by Murakami et al. (1976).

\section{ACTOMYOSIN ATPase ASSAYS}

Porcine cardiac myosin, rabbit skeletal actin and human cardiac Tm and Tns were homogenized to a final concentration of $0.6 \mu \mathrm{M}$ myosin, $3.5 \mu \mathrm{M}$ actin, $1 \mu \mathrm{M} \mathrm{Tm}$, and $0-1.5 \mu \mathrm{M}$ Tn in ATPase assay buffer ( $40 \mathrm{mM} \mathrm{KCl}, 15 \mathrm{mM}$ MOPS pH 7.0, $3.5 \mathrm{mM} \mathrm{MgCl}_{2}$, $1 \mathrm{mM}$ DTT, $1 \mathrm{mM}$ EGTA (pCa 9.5) or $0.416 \mathrm{mM} \mathrm{CaCl}_{2}$ (pCa 4). For Tm titration studies, Tm was varied from 0 to $1.5 \mu \mathrm{M}$, in the absence of Tn. ATPase reactions were initiated with the addition 
of ATP to a final concentration of $2.9 \mathrm{mM}$, the amount calculated to yield approximately $1 \mathrm{mM}$ free $\mathrm{MgCl}_{2}$ (Dweck et al., 2005). ATPase assays were performed at $37^{\circ} \mathrm{C}$ for $20 \mathrm{~min}$ and the reaction was stopped with the addition of $25 \mu \mathrm{L}$ of $35 \%$ trichloroacetic acid. The amount of inorganic phosphate released during the ATPase assays was determined by use of the Fiske-Subbarrow reagent (Fiske and Subbarrow, 1925).

\section{STATISTICAL ANALYSIS}

For all measurements, significance of comparison to ASWT was determined by 1- or 2-way ANOVA followed by Dunnett's multiple comparisons test against ASWT. In all figures significance is denoted as follows: ${ }^{*} p<0.05,{ }^{\dagger} p<0.01,{ }^{\ddagger} p<0.001$.

\section{MUTANT TM MODELING}

To model the effects of the E40K, E54K, and E62Q mutations, the structure of a fragment of Tm containing residues $1-80$, pdb accession number 1IC2 were used as a starting point (Brown et al., 2001). To model the effect of the mutation L185R the X-ray structure of a mid-region fragment of Tm, pdb accession number 2B9C was used as a starting point (Brown et al., 2005). In all cases, the point mutations were introduced into the respective crystal structures, hydrogens and Gastiger- Huckel charges were added to the structure, and the structures were then minimized with unrestrained helices to convergence using the program Sybyl (Tripos Associates). The molecular graphics images were produced using the UCSF Chimera package from the Computer Graphics Laboratory, University of California, San Franscisco (Pettersen et al., 2004) supported by National Institutes of Health grant P41 RR-01081.

\section{RESULTS}

\section{EFFECTS OF MUTATIONS ON TN CONCENTRATION DEPENDENT ACTIVATION AND INHIBITION OF ACTOMYOSIN ATPase ACTIVITY}

To investigate the effects of the mutations on actin-myosin interaction and thin filament regulation of ATPase activity, the actin and myosin concentrations were held constant and the effects of increasing concentrations of Tn on actomyosin ATPase rates were measured. As a control for the assay, a well-characterized HCM mutation E180G was also included in the actomyosin ATPase assays. The results of these studies show that the two DCM mutants gave strikingly different results where E40K causes a significant decrease in maximal ATPase activity and E54K causes an increase (Figure 1A). Increased rates over ASWT were measured at high $\left[\mathrm{Ca}^{2+}\right]$ for all three HCM mutants (Figure 1A).

In all assays, ATPase rates reached a maximum by $1 \mu \mathrm{M}$ Tn. Therefore, relative maximal actomyosin ATPase rates of DCM (Figure 1B) and HCM (Figure 1C) mutant Tm samples in high and low $\left[\mathrm{Ca}^{2+}\right]$ at $1 \mu \mathrm{M}$ Tm and $1-1.5 \mu \mathrm{M}$ Tn, were plotted to better illustrate the $\left[\mathrm{Ca}^{2+}\right]$-dependent trends. When normalized to ASWT in high $\left[\mathrm{Ca}^{2+}\right]$, the rate at low $\left[\mathrm{Ca}^{2+}\right]$ is $8.5 \pm 0.3 \%$. Consistent with plots in Figure 1A, the maximal rates for $\mathrm{E} 40 \mathrm{~K}$ in high and low $\left[\mathrm{Ca}^{2+}\right]$ were lower: $85 \pm 1.8 \%$ and $7.1 \pm 0.1 \%$, respectively. The maximal rates for E54K in high and low $\left[\mathrm{Ca}^{2+}\right]$ were higher: $122 \pm 3.8 \%$ and $9.9 \pm 0.4 \%$, respectively. All HCM associated mutants showed significantly increased rates in high $\left[\mathrm{Ca}^{2+}\right]$, of $122 \pm 1.7 \%$ for E62Q, $112 \pm 2.9 \%$ for $\mathrm{E} 180 \mathrm{G}$, and
$117 \pm 2.1 \%$ for L185R. In low $\left[\mathrm{Ca}^{2+}\right]$, all but E62Q had significantly increased rates: $8.7 \pm 0.3 \%$ for E62Q, $12 \pm 0.5 \%$ for E180G, and $11 \pm 0.5 \%$ for L185R.

In addition to the differences in maximal $\mathrm{Ca}^{2+}$-dependent activity, the rates in the absence of Tn vary significantly for some mutants, suggesting that $\mathrm{Tm}$ alone is affecting the actomyosin ATPase rate (Figure 1A). In the absence of Tn, the actomyosin ATPase activity is not $\mathrm{Ca}^{2+}$-dependent. Comparison of averaged baseline values in the absence of Tn showed that the ATPase activity in $\mathrm{mol} \mathrm{Pi} / \mathrm{mol} \mathrm{myosin} / \mathrm{sec}$ was $0.27 \pm 0.01$ for ASWT, and significantly lower at $0.23 \pm 0.1$ for E40K. The baseline activity was significantly higher for E54K and E62Q, which were both $0.32 \pm 0.1$. The baseline activity differences were not statistically significant for E62Q and E180G, which were $0.28 \pm 0.02$ and $0.30 \pm 0.01$

\section{EFFECTS OF THE MUTATIONS ON THE TM CONCENTRATION DEPENDENT INHIBITION OF ACTOMYOSIN ATPase ACTIVITY}

Increasing the concentration of Tm is known to result in diminished actomyosin ATPase activity, when the myosin concentration is held low and constant (Lehrer and Morris, 1982). To gain information on the possible effects of the mutations on Tm binding to actin and consequently, inhibition of actin-myosin interactions, actomyosin ATPase rates were measured at low $\left[\mathrm{Ca}^{2+}\right]$ conditions with increasing amounts of $\mathrm{Tm}$, in the absence of Tn (Figure 2A). Comparison of the Tm concentration dependent inhibition showed that E40K inhibited actomyosin ATPase activity better than ASWT, while E54K did not inhibit the actomyosin ATPase activity at all (Figure 2A). HCM associated mutants did not inhibit the actomyosin ATPase activity over ASWT, except the E62Q which increased the actomyosin ATPase activity (Figure 2A). Ratios of protein mixtures for ASWT and mutants were confirmed by Coomassie gel analysis (Figure 2B) and activity rates at $1 \mu \mathrm{M}$ Tm compared for all mutants (Figure 2C). Consistent with trends seen in the Tm titration studies, and the baseline rate differences measured in the Tn titration studies, the rates with $1 \mu \mathrm{M}$ Tm are significantly lower in E40K and significantly higher in E54K and E62Q mutants. The rates of E180G and L185R are increased but not significantly.

\section{EFFECTS OF MUTATIONS ON TM STRUCTURE}

The effects of the mutations investigated by CD spectra measurements showed that the single point mutations do not have significant effects on the mean residue ellipticity of Tm. Minor differences were seen, but are not significant (data not shown). Further investigation of the effects of the mutations on Tm structure by thermal denaturation showed interestingly, that the point mutations have differential effects on the melting transitions of Tm (Figure 3A). The individual denaturation curves were fitted to a three-transition model to identify the melting transition temperatures, the enthalpy change associated with unfolding and the fraction of the change in ellipticity due to each transition. The values are summarized in Table $\mathbf{1}$. In three of the cases, the results of the mutations caused the appearances of an additional transition, suggesting that the changes were local, and affecting a relatively small portion of the Tm molecule (Figure 3B, Table 1). To assess the effects of the mutations on Tm stability, $\mathrm{T}_{\mathrm{M} 50}$ values were 


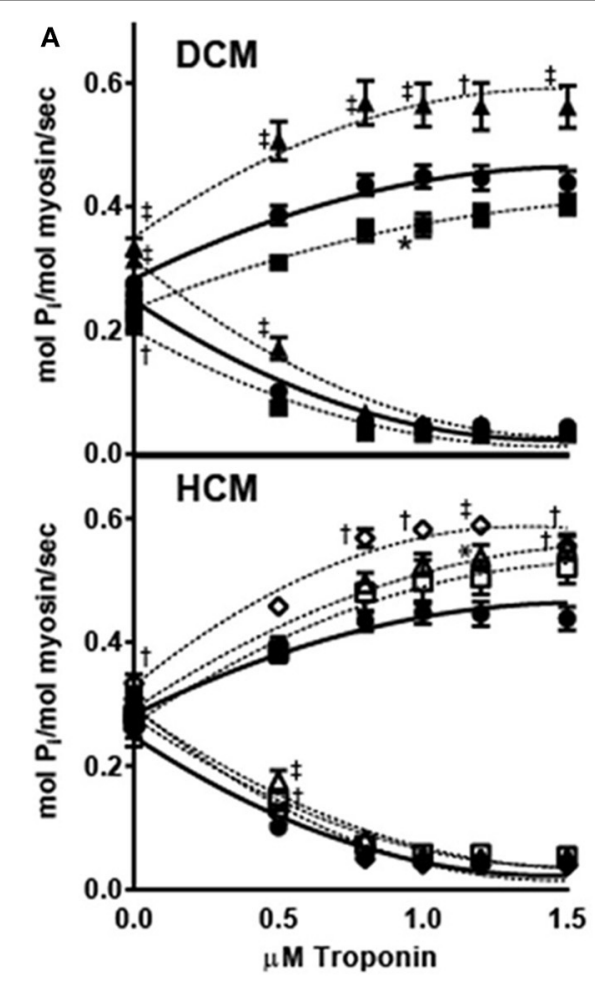

FIGURE 1 | Effects of DCM and HCM Tm mutants on Tn concentration dependent actomyosin ATPase activity. (A) The Tn concentrationdependent ATPase rates of actomyosin samples prepared with ASWT and mutant Tms were compared. Tn was titrated from 0 to $1.5 \mu \mathrm{M}$ into mixtures of $0.6 \mu \mathrm{M}$ myosin, $3.5 \mu \mathrm{M}$ actin, and $1 \mu \mathrm{M} \mathrm{Tm}$, and the actomyosin ATPase activation at high ( $\mathrm{pCa} 4$ ) $\left[\mathrm{Ca}^{2+}\right]$ (up-sloping) and inhibition at low (pCa 9.5) $\left[\mathrm{Ca}^{2+}\right]$ (down-sloping) were measured for DCM (upper panel) and $\mathrm{HCM}$ (lower panel) associated mutations. The results represent the mean \pm SE of calculated activity rates of experiments $(n=5)$ performed in triplicate. Samples graphed on DCM panel: ASWT (filled circle), E40K (filled square),
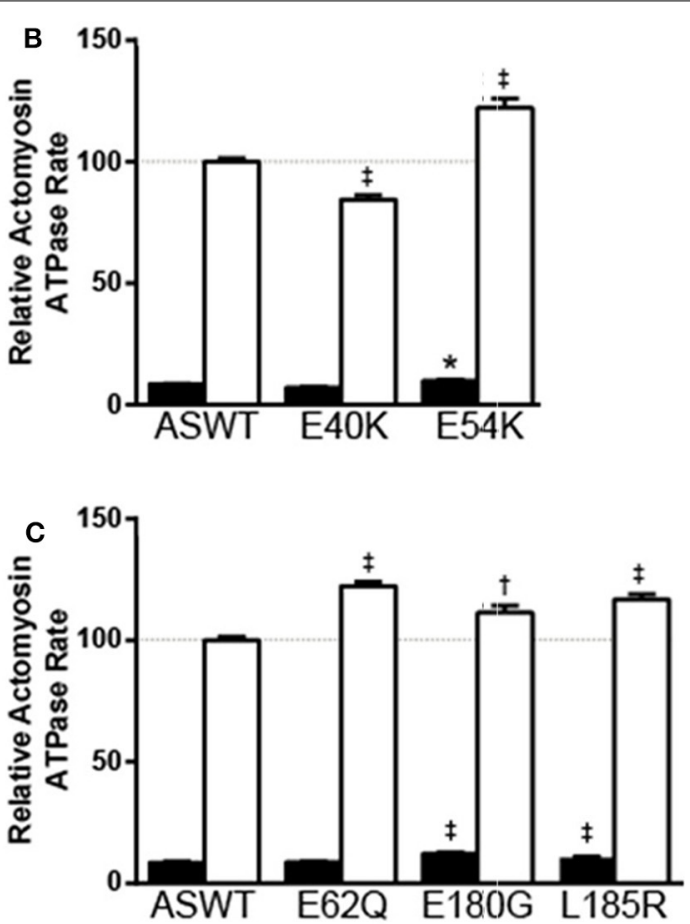

E54K (filled triangle); on HCM panel: ASWT (filled circle), E620 (open diamond), E180G (open square), L185R (open triangle). The maximal ATPase activity values of ASWT and DCM-associated (B) and HCM-associated (C) mutant Tm reconstituted actomyosin solutions in low (pCa 9.5; filled bar) and high (pCa 4; open bar) $\left[\mathrm{Ca}^{2+}\right]$ were compared. Relative rates shown graphed are a percentage of the mean high $\left[\mathrm{Ca}^{2+}\right]$ activated ASWT rate obtained from mixtures of $0.6 \mu \mathrm{M}$ myosin, $3.5 \mu \mathrm{M}$ actin, $1 \mu \mathrm{M} \mathrm{Tm}$, and $1-1.5 \mu \mathrm{M}$ Tn. Mean percentages \pm SE were graphed; $n=20$. Statistical significance were determined by ANOVA followed by Dunnett's multiple comparisons test; ${ }^{*} p<0.05,{ }^{\dagger} p<0.01,{ }^{\ddagger} p<0.001$. calculated from the thermal denaturation curves of ASWT and mutants. The $\mathrm{T}_{\mathrm{M} 50}$ values show shifts temperatures suggestive of decreased stability for the DCM E40K mutant and increased stability for E62Q and L185R (Figure 3C). The stability for the E54K mutant did not change compared to ASWT (Figure 3C, Table 1).

Changes in Tm stability were explored through energy minimized models of the Tm mutations, which gives insight into the potential effects at the dimer interface (Figure 4). The heptad repeat position of the native protein residues and the expected interactions within the Tm molecule interface has been described in detail (Brown and Cohen, 2005). In the native protein, E40 in position $e$ has a strong inter-chain interaction with R35 in position $g$, where the carboxyl group of E40 can interact with the amine side-chains of R35 (Brown et al., 2001, 2005). Destabilization of the coiled-coil by E40K is observed. In addition, E54 displays an intra-chain i to i+3 interactions with K51 and an inter-chain interaction with $\mathrm{K} 49$ in position $g$. Destabilization of the coiled-coil by E54K mutation is observed. Mutation of E62 to glutamine would not disrupt its interaction with K59 since the two could still form a strong hydrogen bond, and would allow interaction of the amide nitrogen with the carboxyl of D58, which could result in stabilization of the molecule. However, the carboxyl groups of E62 (carbon atoms in cyan) have intra-chain interactions with the amine groups of K65 (carbon atoms in magenta). When the E62 is mutated to Q, additional interactions occur; the carboxyl group of the amide side-chain of Q62 can still interact with the amine side-chain of K65 and the carboxyl groups of D58 (carbon atoms in coral) can now hydrogen bond to the amide hydrogens of the Q62 side chain. L185 is a hydrophobic residue that is exposed to the solvent and does not have any close inter- or intra-chain interactions. The side-chains of L185 and R185 do not interact with K189 (carbon atoms in magenta), but when L185 (carbon atoms in cyan) is mutated to R, it loses the entropic penalty of having a hydrophobic residue exposed to the solvent, and the basic side-chain can interact with the carboxyl groups of E181 (carbon atoms in coral), stabilizing the molecule.

\section{DISCUSSION}

The data presented here reports on the effects of various cardiomyopathy associated mutations on Tm structure and function. In the investigation of the effects of the mutations on Tndependent activation and inhibition of actomyosin ATPase activity, the results show that the effects of the HCM-associated mutations on the thin filament segregate well with disease, increasing 


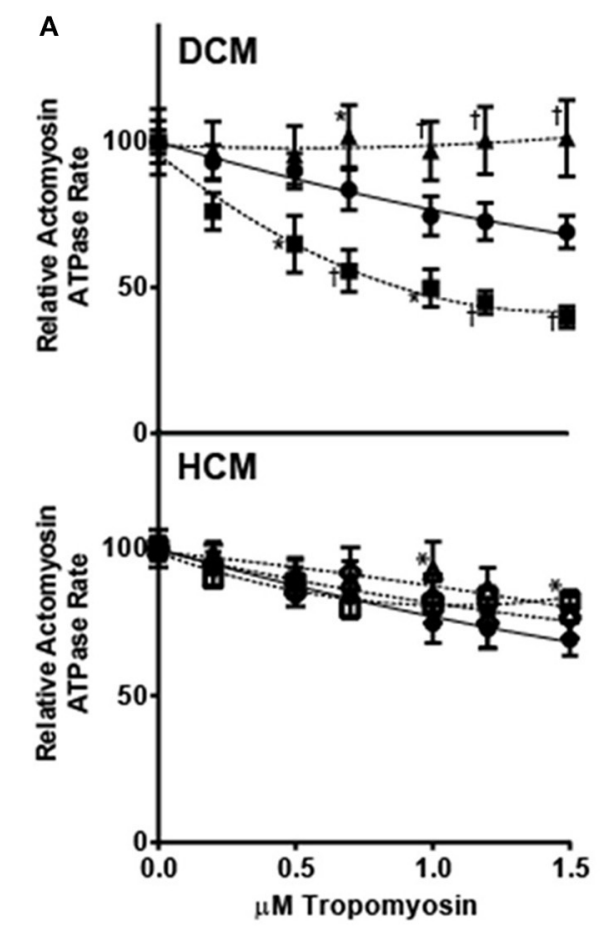

FIGURE 2 | Effects of DCM and HCM-associated mutations on Tm-dependent inhibition of actomyosin ATPase activity. (A) The effects of Tm concentration dependent inhibition of actomyosin ATPase activity were compared between ASWT and DCM (upper panel) and HCM (lower panel) associated mutants. Tm was titrated from 0 to $1.5 \mu \mathrm{M}$ into solutions of $0.6 \mu \mathrm{M}$ myosin and $3.5 \mu \mathrm{M}$ actin, in low $\mathrm{Ca}^{2+}$ (pCa 9.5) buffer, and the actomyosin ATPase activity measured. The results represent mean \pm SE of activity rates normalized to the maximum ATPase activity at $0 \mu \mathrm{M} \mathrm{Tm}$. All samples were measured in triplicate; $n=5$. Samples graphed on DCM panel: ASWT (filled circle),
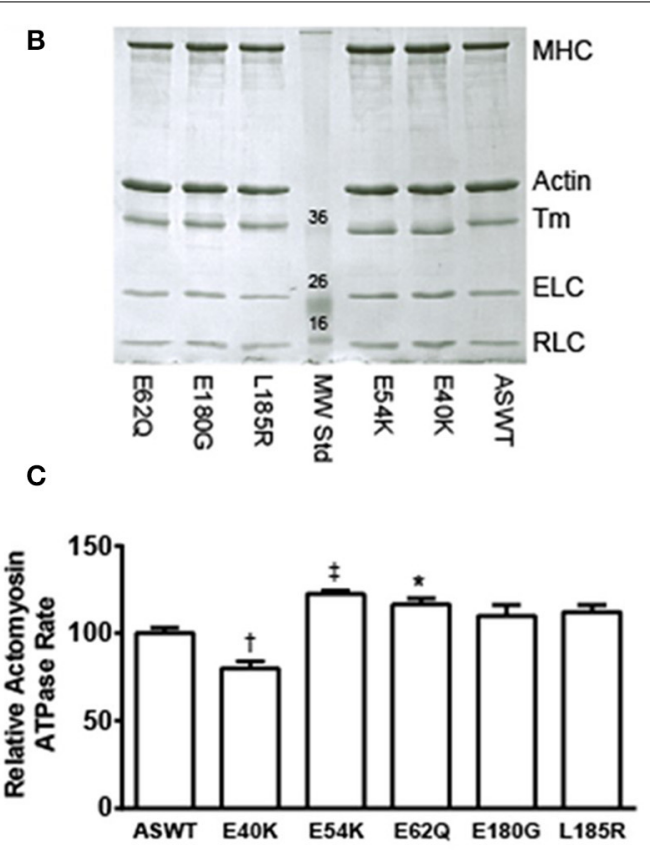

E40K (filled square), E54K (filled triangle); on HCM panel: ASWT (filled circle), E620 (open diamond), E180G (open square), L185R (open triangle). (B) Coomassie stained SDS-PAGE analysis of Tm titration study samples. Samples taken from actomyosin ATPase activity assay mixtures $(0.6 \mu \mathrm{M}$ myosin, $3.5 \mu \mathrm{M}$ actin, $1 \mu \mathrm{M}$ Tm) were run on a $12 \%$ SDS-PAGE gel, to confirm purity and proper ratios of proteins utilized in the Tm titration studies. (C) Comparison of relative ATPase rates in the presence of $1 \mu \mathrm{M} \mathrm{Tm}$. All samples were measured in triplicate; $n=16$. Statistical significance were determined by ANOVA followed by Dunnett's multiple comparisons test; ${ }^{*} p<0.05,{ }^{\dagger} p<0.01,{ }^{\ddagger}{ }_{p}<0.001$. maximal actomyosin ATPase rates (Figure 1C), as previously reported by myofibrillar ATPase assays (Chang et al., 2005). Structurally, the position of the HCM-associated mutation E62Q is an actin binding site residue (Phillips et al., 1986), and energy minimized structural models predicted an increase in Tm dimer stability, which is confirmed by the thermal denaturation study presented here (Figure 3C). Due to its position in the Tm coiledcoil, E62 does not have any inter-chain interactions in either the WT or mutant proteins. The position of the HCM-associated mutation L185R is also a consensus actin binding residue, and is in a region of $\mathrm{Tm}$ found to be critical for actin binding and regulation (Greenfield et al., 2002; Singh and Hitchcock-Degregori, 2006). Although there are local stability changes associated with E62Q which are not seen in L185R (Figure 3B), both mutations caused increases in overall stability of the $\mathrm{Tm}$ molecule (Figure 3C). Energy minimized model of the L185R mutant structure suggests the reason for stabilization of $\mathrm{Tm}$ consistent with increased $\mathrm{T}_{M 50}$ of thermal denaturation (Figure 3C).

Consistent with our findings, several studies have reported that changes in Tm stability and consequently disinhibition of the myofilament at low $\left[\mathrm{Ca}^{2+}\right]$ and increasing the number of active crossbridges is one of the mechanisms by which Tm mutations associated with HCM may induce diastolic dysfunction (Li et al., 2012; Ly and Lehrer, 2012). In terms of clinical manifestation, both HCM associated mutations were found in families which were greatly affected by the mutation as demonstrated by the segregation studies (Van Driest et al., 2002; Jongbloed et al., 2003). The L185R mutation has only been investigated by our group (Chang et al., 2005), and the recent identification of this mutation in several members of another family with HCM, including an 8-year-old girl who had sudden cardiac death (Makhoul et al., 2011), underscores the need for characterization of the molecular mechanisms affected by Tm mutations.

When the effects of the mutations are measured by myofibrillar ATPase assays, both DCM-associated mutations E40K and E54K had no effect on the inhibition of ATPase activity (Chang et al., 2005). Examination of the current Tn titration study shows that the baseline rates in the absence of Tn are different from ASWT for E40K and E54K (Figure 1A). While E40K has diminished activity in low $\left[\mathrm{Ca}^{2+}\right]$, E54K has a slight increase (Figure 1B), in agreement with the results of the Tm titration study (Figure 2C). The highly increased inhibition of actomyosin activity by E40K and total lack of inhibition by E54K (Figure 2A), is suggestive of distinct mechanisms by which these two mutations cause DCM. 

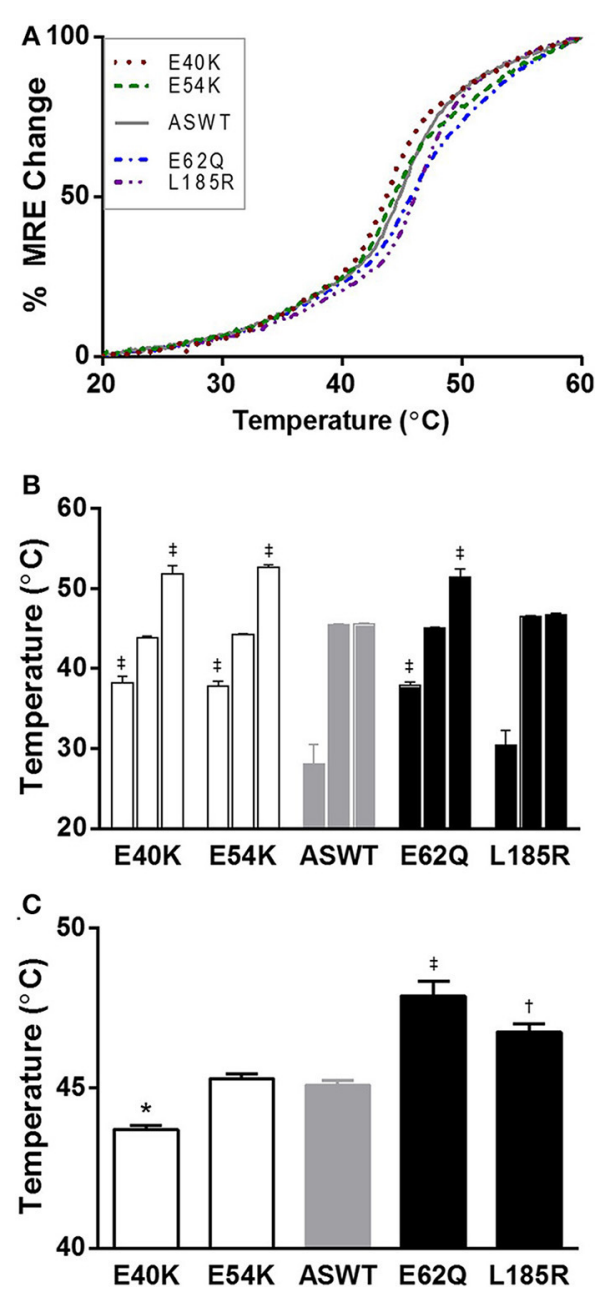

FIGURE 3 | Thermal denaturation spectra of ASWT and mutants. (A) Purified Tms were denatured by increasing the temperature from 20 to $60^{\circ} \mathrm{C}$, and the changes in ellipticity measured at each $0.2^{\circ} \mathrm{C}$. Each spectra represents averaged mean residue ellipticity (MRE) of $n=9$. (B) Thermal transition temperatures obtained from fitting the denaturation spectra to three transition models are shown as mean values \pm s.e.m. (C) Half maximal denaturation temperatures for ASWT and mutants are shown as mean values \pm s.e.m. Statistical significance were determined by ANOVA followed by Dunnett's multiple comparisons test; ${ }^{*} p<0.05,{ }^{\dagger} p<0.01,{ }^{\ddagger} p<0.001$.
Furthermore, the in vitro data for the E54K mutation correlate well with the in vivo phenotype reported in a transgenic mouse. The transgenic mice expressing the Tm E54K mutation demonstrated impaired systolic and diastolic functions (Rajan et al., 2007). The decreased $\mathrm{Ca}^{2+}$ sensitivity of the myofilament can be associated with the systolic dysfunction, while an inability of the heart to relax correlates with the solutions studies where the E54K does not inhibit the actomyosin ATPase (Figure 2A). This result does not agree with general findings reported for DCM-associated thin filament mutations, such as decreased $\mathrm{Ca}^{2+}$ sensitivity of contraction and decreased maximal force in reconstituted cardiac muscle fibers (Chang and Potter, 2005; Mirza et al., 2005; Willott et al., 2010), but is in agreement with previous studies by our group and others (Chang et al., 2005; Mirza et al., 2005, 2007). The possibility of the effects of E54K on actomyosin ATPase assays being an artifact or miscalculation is ruled out based on its agreement with other groups' results, and the fact that SDSPAGE analysis shows that E54K Tm is present, and the amount of myosin is similar for all samples (Figure 2B).

Based on the sequence of Tm, and the crystal structures of Tm fragments (Brown et al., 2001, 2005), the following interactions may be affected by the mutations, and contributing to the differences in thermal stability. The mutation E40K occurs directly after a very unstable region of the Tm, which has alanines, lysines and serines in the $a$ and $d$ positions of the coiled coil interface, comprising a highly flexible region (Phillips, 1986). Like E40, E54 is an external glutamate which is postulated by Phillips to be a "consensus" actin binding residue (Phillips, 1986; Phillips et al., 1986). As postulated, both mutations E40K and E54K have been shown to reduce the affinity of $\mathrm{Tm}$ for actin, although at different thin filament states (On and Off states, respectively) (Mirza et al., 2007). Based on the actomyosin ATPase assay Tm titration studies, rather than substantially affecting the actin binding affinity (Mirza et al., 2007), the mutations may be affecting the position of Tm on actin itself, altering the blocked or open position of Tm on actin (Orzechowski et al., 2014). Indeed, the two mutations have been shown to affect the transition from weakly- to strongly-bound crossbridges when reconstituted into muscle fibers from which endogenous Tm was removed (Borovikov et al., 2009b, 2011). In the case of $\mathrm{E} 40 \mathrm{~K}$, the position of Tm on actin may be blocking more of the myosin interaction sites than ASWT and thereby diminishing the baseline activity to a greater extent than ASWT.

Table 1 | Thermal transition temperatures of ASWT and mutants.

\begin{tabular}{|c|c|c|c|c|c|c|c|c|c|c|c|}
\hline Mutant & $\mathbf{T}_{\mathbf{M} 50}$ & $\mathrm{~T}_{\mathrm{M1}}$ & $\mathrm{T}_{\mathrm{M} 2}$ & $\mathrm{~T}_{\mathrm{M3}}$ & $\Delta \mathrm{H}_{1}$ & $\Delta \mathrm{H}_{2}$ & $\Delta \mathrm{H}_{3}$ & $\varepsilon_{1}$ & $\varepsilon_{2}$ & $\varepsilon_{3}$ & $n$ \\
\hline ASWT & $45.3 \pm 0.3$ & $28.1 \pm 2.5$ & $\mathrm{n} / \mathrm{a}$ & $45.5 \pm 0.1$ & $-22 \pm 8$ & $\mathrm{n} / \mathrm{a}$ & $-144 \pm 29$ & $0.38 \pm 0.03$ & $\mathrm{n} / \mathrm{a}$ & $0.64 \pm 0.04$ & 9 \\
\hline E54K & $45.3 \pm 0.1$ & $37.8 \pm 0.6^{\ddagger}$ & $44.3 \pm 0.1$ & $52.6 \pm 0.3^{\ddagger}$ & $-36 \pm 10$ & $-146 \pm 23$ & $-118 \pm 22$ & $0.39 \pm 0.07$ & $0.36 \pm 0.06$ & $0.24 \pm 0.05$ & 9 \\
\hline E62O & $47.9 \pm 0.5^{\ddagger}$ & $37.9 \pm 0.4^{\ddagger}$ & $45.1 \pm 0.1$ & $51.5 \pm 1.0^{\ddagger}$ & $-30 \pm 7$ & $-149 \pm 64$ & $-141 \pm 48$ & $0.35 \pm 0.09$ & $0.35 \pm 0.11$ & $0.29 \pm 0.19$ & 11 \\
\hline
\end{tabular}

Shown are the results of thermal denaturation spectra fitted to a two and three-transition model (see methods for description). The values represent mean \pm SE of

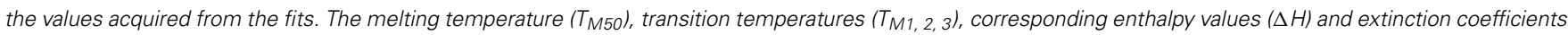
( $\varepsilon$ ) for each mutant are reported for the spectra measured the number of times shown (n). Statistical significance for temperatures were determined by ANOVA followed by Dunnett's multiple comparisons test; ${ }^{*} p<0.05,{ }^{\ddagger} p<0.001$. 


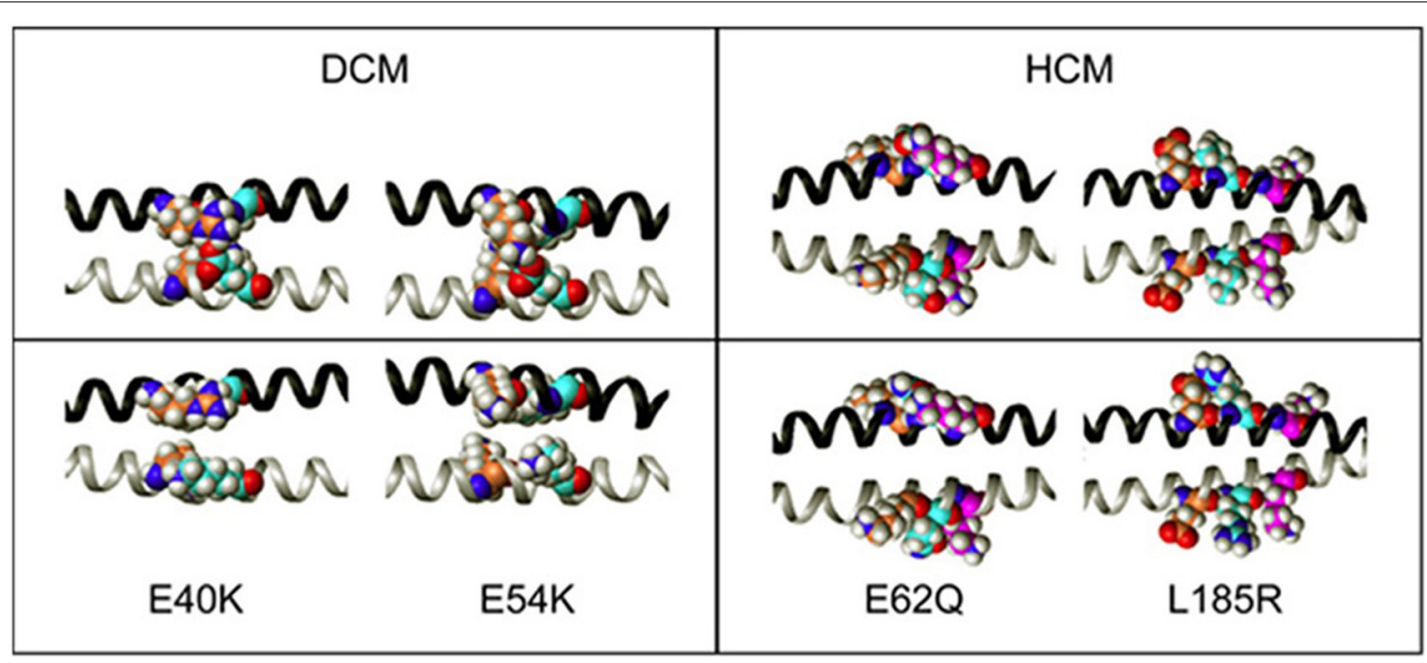

FIGURE 4 | The effects of DCM and HCM-associated mutations on the side-chain interactions of $\mathrm{Tm}$ dimers. Side-chain interactions were modeled by Sybyl, and compared with the corresponding wildtype (upper half) for each mutation indicated. In all the panels the nitrogen atoms are blue and the oxygen atoms are red. The helical chairs are depicted as ribbons, with one chain of Tm in black and the other chain in gray.
The decrease in maximal activity was not seen in the previously published myofibrillar ATPase assays (Chang et al., 2005), but based on the Tn and Tm ATPase inhibition studies presented here, obstruction of the myosin binding sites on actin likely exists, and this effect may be diminished in myofibrils due to the more intact nature of the system. In actomyosin ATPase assays, the effects of $\mathrm{Tm}$ alone on actomyosin interaction are masked by the presence of $\mathrm{Tn}$, which confers $\mathrm{Ca}^{2+}$ sensitivity and increases the rate beyond the extent inhibited by $\mathrm{Tm}$. In the case of E54K, Tn alone does not fully regulate the actomyosin ATPase activity, as evidenced by the increased activity over ASWT (Figure 1A), which is in agreement with another study, where increased cooperative unit size of actin-Tm and actin-Tm-Tn caused by E54K, was contrasted to a decrease caused by $\mathrm{E} 40 \mathrm{~K}$, despite comparative phenotype (Olson et al., 2001) and decreases in $\mathrm{Ca}^{2+}$ sensitivity in more intact systems (Chang et al., 2005; Mirza et al., 2005). In a more intact system such as in myofibrils (Chang et al., 2005), where spatial organization of the thick and thin filaments are maintained, the mutation may be further diminishing the ATPase activity, possibly to the same extent of $\mathrm{E} 40 \mathrm{~K}$, thus producing similar effects on contractility.

The results of the thermal denaturation studies suggest that a single point mutation does affect the global Tm structure, albeit not the total helical content, but the melting transitions of the Tm dimer itself. Characterization of these regions in Tm have shown that a denaturation curve of $\mathrm{Tm}$, like those shown in Figure 3, is comprised of numerous overlapping melting transitions which correspond to flexible sequences that are essential for actin binding (Greenfield and Hitchcock-Degregori, 1995). The lack of significant differences in the secondary structure of $\mathrm{Tm}$ and the local destabilizations found in the denaturation curves suggest that the mutation induced alterations occur at the level of intraand inter-coil interactions rather than at the level of the whole $\mathrm{Tm}$ dimer. However, when in complex with $\mathrm{Tn}$, local instabilities in $\mathrm{Tm}$ are known to have long-range effects on the overlapping ends of Tm (Mamidi et al., 2013a,b). In conclusion, the findings reported here show that the structural alterations in Tm observed, may affect the position of Tm on actin, which in solution may alter the inhibitory or activating states of the thin filament. The results of the studies conducted suggest that in agreement with the wide range of clinical phenotypes, the mutational effects on structure and protein-protein interactions vary between and within disease categories.

\section{ACKNOWLEDGMENTS}

This work was supported by National Institutes of Health Grants HL42325 and HL67415 to James D. Potter. Norma Greenfield and Abhishek Singh's contributions were supported by $\mathrm{NIH}$ grant, GM-36326, to Sarah E. Hitchcock-DeGregori and an NIH Interdisciplinary Research Workforce fellowship to Abhishek Singh. Jose R. Pinto acknowledges the support from the FSU College of Medicine. The content of this study is solely the responsibility of the authors, and does not necessarily represent the official view of the awarding organization. Authors thank Dr. Sarah E. Hitchcock-DeGregori of Robert Wood Johnson Medical School, Rutgers University, for the critical reading of the manuscript and helpful suggestions.

\section{REFERENCES}

Bai, F., Groth, H. L., and Kawai, M. (2012). DCM-related tropomyosin mutants E40K/E54K over-inhibit the actomyosin interaction and lead to a decrease in the number of cycling cross-bridges. PLOS ONE 7:e47471. doi: 10.1371/journal.pone.0047471

Barua, B., Fagnant, P. M., Winkelmann, D. A., Trybus, K. M., and HitchcockDegregori, S. E. (2013). A periodic pattern of evolutionarily conserved basic and acidic residues constitutes the binding interface of actin-tropomyosin. J. Biol. Chem. 288, 9602-9609. doi: 10.1074/jbc.M113.451161

Borovikov, Y. S., Avrova, S. V., Karpicheva, O. E., Robinson, P., and Redwood, C. S. (2011). The effect of the dilated cardiomyopathy-causing Glu40Lys TPM1 mutation on actin-myosin interactions during the ATPase cycle. Biochem. Biophys. Res. Commun. 411, 496-500. doi: 10.1016/j.bbrc.2011.06.138

Borovikov, Y. S., Karpicheva, O. E., Avrova, S. V., and Redwood, C. S. (2009a). Modulation of the effects of tropomyosin on actin and myosin conformational 
changes by troponin and $\mathrm{Ca}^{2+}$. Biochim. Biophys. Acta 1794, 985-994. doi: 10.1016/j.bbapap.2008.11.014

Borovikov, Y. S., Karpicheva, O. E., Avrova, S. V., Robinson, P., and Redwood, C. S. (2009b). The effect of the dilated cardiomyopathy-causing mutation Glu54Lys of alpha-tropomyosin on actin-myosin interactions during the ATPase cycle. Arch. Biochem. Biophys. 489, 20-24. doi: 10.1016/j.abb.2009. 07.018

Borovikov, Y. S., Karpicheva, O. E., Chudakova, G. A., Robinson, P., and Redwood, C. S. (2009c). Dilated cardiomyopathy mutations in alpha-tropomyosin inhibit its movement during the ATPase cycle. Biochem. Biophys. Res. Commun. 381, 403-406. doi: 10.1016/j.bbrc.2009.02.054

Brown, J. H., and Cohen, C. (2005). Regulation of muscle contraction by tropomyosin and troponin: how structure illuminates function. Adv. Protein Chem. 71, 121-159. doi: 10.1016/S0065-3233(04)71004-9

Brown, J. H., Kim, K. H., JUN, G., Greenfield, N. J., Dominguez, R., Volkmann, N., et al. (2001). Deciphering the design of the tropomyosin molecule. Proc. Natl. Acad. Sci. U.S.A. 98, 8496-8501. doi: 10.1073/pnas.131219198

Brown, J. H., Zhou, Z., Reshetnikova, L., Robinson, H., Yammani, R. D., Tobacman, L. S., et al. (2005). Structure of the mid-region of tropomyosin: bending and binding sites for actin. Proc. Natl. Acad. Sci. U.S.A. 102, 18878-18883. doi: 10.1073/pnas.0509269102

Chang, A. N., Harada, K., Ackerman, M. J., and Potter, J. D. (2005). Functional consequences of hypertrophic and dilated cardiomyopathy-causing mutations in alpha-tropomyosin. J. Biol. Chem. 280, 34343-34349. doi: 10.1074/jbc.M505014200

Chang, A. N., and Potter, J. D. (2005). Sarcomeric protein mutations in dilated cardiomyopathy. Heart Fail. Rev. 10, 225-235. doi: 10.1007/s10741-005-5252-6

Dweck, D., Reyes-Alfonso, A. Jr., and Potter, J. D. (2005). Expanding the range of free calcium regulation in biological solutions. Anal. Biochem. 347, 303-315. doi: 10.1016/j.ab.2005.09.025

Farah, C. S., and Reinach, F. C. (1995). The troponin complex and regulation of muscle contraction. Faseb. J. 9, 755-767.

Fiske, C. H., and Subbarrow, Y. (1925). The colorimetric determination of phosphorous. J. Biol. Chem. 66, 375-400.

Force, T., Bonow, R. O., Houser, S. R., Solaro, R. J., Hershberger, E., Adhikari, B., et al. (2010). Research priorities in hypertrophic cardiomyopathy: report of a working group of the national heart, lung, and blood institute. Circulation 122, 1130-1133. doi: 10.1161/CIRCULATIONAHA.110.950089

Gomes, A. V., Guzman, G., Zhao, J., and Potter, J. D. (2002). Cardiac troponin $\mathrm{T}$ isoforms affect the $\mathrm{Ca}^{2+}$ sensitivity and inhibition of force development. Insights into the role of troponin T isoforms in the heart. J. Biol. Chem. 277, 35341-35349. doi: 10.1074/jbc.M204118200

Gordon, A. M., Homsher, E., and Regnier, M. (2000). Regulation of contraction in striated muscle. Physiol. Rev. 80, 853-924.

Greenfield, N. J. (2006). Using circular dichroism collected as a function of temperature to determine the thermodynamics of protein unfolding and binding interactions. Nat. Protoc. 1, 2527-2535. doi: 10.1038/nprot.2006.204

Greenfield, N. J., and Hitchcock-Degregori, S. E. (1995). The stability of tropomyosin, a two-stranded coiled-coil protein, is primarily a function of the hydrophobicity of residues at the helix-helix interface. Biochemistry 34 , 16797-16805. doi: 10.1021/bi00051a030

Greenfield, N. J., Palm, T., and Hitchcock-Degregori, S. E. (2002). Structure and interactions of the carboxyl terminus of striated muscle alpha-tropomyosin: it is important to be flexible. Biophys. J. 83, 2754-2766. doi: 10.1016/S00063495(02)75285-5

Hammell, R. L., and Hitchcock Degregori, S. E. (1997). The sequence of the alternatively spliced sixth exon of alpha-tropomyosin is critical for cooperative actin binding but not for interaction with troponin. J. Biol. Chem. 272, 22409-22416. doi: $10.1074 /$ jbc. 272.36 .22409

Hershberger, R. E., Hedges, D. J., and Morales, A. (2013). Dilated cardiomyopathy: the complexity of a diverse genetic architecture. Nat. Rev. Cardiol. 10, 531-547. doi: 10.1038/nrcardio.2013.105

Holroyde, M. J., Robertson, S. P., Johnson, J. D., Solaro, R. J., and Potter, J. D. (1980). The calcium and magnesium binding sites on cardiac troponin and their role in the regulation of myofibrillar adenosine triphosphatase. J. Biol. Chem. 255, 11688-11693.

Jongbloed, R. J., Marcelis, C. L., Doevendans, P. A., Schmeitz-Mulkens, J. M., Van Dockum, W. G., Geraedts, J. P., et al. (2003). Variable clinical manifestation of a novel missense mutation in the alpha-tropomyosin (TPM1) gene in familial hypertrophic cardiomyopathy. J. Am. Coll. Cardiol. 41, 981-986. doi: 10.1016/S0735-1097(02)03005-X

Landis, C. A., Bobkova, A., Homsher, E., and Tobacman, L. S. (1997). The active state of the thin filament is destabilized by an internal deletion in tropomyosin. J. Biol. Chem. 272, 14051-14056. doi: 10.1074/jbc.272.22.14051

Landis, C., Back, N., Homsher, E., and Tobacman, L. S. (1999). Effects of tropomyosin internal deletions on thin filament function. J. Biol. Chem. 274, 31279-31285. doi: 10.1074/jbc.274.44.31279

Lehrer, S. S., and Morris, E. P. (1982). Dual effects of tropomyosin and troponintropomyosin on actomyosin subfragment 1 ATPase. J. Biol. Chem. 257, 8073-8080.

Li, X. E., Suphamungmee, W., Janco, M., Geeves, M. A., Marston, S. B., Fischer, S., et al. (2012). The flexibility of two tropomyosin mutants, D175N and E180G, that cause hypertrophic cardiomyopathy. Biochem. Biophys. Res. Commun. 424, 493-496. doi: 10.1016/j.bbrc.2012.06.141

Ly, S., and Lehrer, S. S. (2012). Long-range effects of familial hypertrophic cardiomyopathy mutations E180G and D175N on the properties of tropomyosin. Biochemistry 51, 6413-6420. doi: 10.1021/bi3006835

Makhoul, M., Ackerman, M. J., Atkins, D. L., and Law, I. H. (2011). Clinical spectrum in a family with tropomyosin-mediated hypertrophic cardiomyopathy and sudden death in childhood. Pediatr. Cardiol. 32, 215-220. doi: 10.1007/s00246010-9843-1

Mamidi, R., Michael, J. J., Muthuchamy, M., and Chandra, M. (2013a). Interplay between the overlapping ends of tropomyosin and the $\mathrm{N}$ terminus of cardiac troponin $\mathrm{T}$ affects tropomyosin states on actin. Faseb. J. 27, 3848-3859. doi: 10.1096/fj.13-232363

Mamidi, R., Muthuchamy, M., and Chandra, M. (2013b). Instability in the central region of tropomyosin modulates the function of its overlapping ends. Biophys. J. 105, 2104-2113. doi: 10.1016/j.bpj.2013.09.026

McLachlan, A. D., and Stewart, M. (1976). The 14-fold periodicity in alphatropomyosin and the interaction with actin. J. Mol. Biol. 103, 271-298. doi: 10.1016/0022-2836(76)90313-2

Mirza, M., Marston, S., Willott, R., Ashley, C., Mogensen, J., McKenna, W., et al. (2005). Dilated cardiomyopathy mutations in three thin filament regulatory proteins result in a common functional phenotype. J. Biol. Chem. 280, 28498-28506. doi: 10.1074/jbc.M412281200

Mirza, M., Robinson, P., Kremneva, E., Copeland, O., Nikolaeva, O., Watkins, H., et al. (2007). The effect of mutations in alpha-tropomyosin (E40K and E54K) that cause familial dilated cardiomyopathy on the regulatory mechanism of cardiac muscle thin filaments. J. Biol. Chem. 282, 13487-13497. doi: 10.1074/jbc.M701071200

Murakami, U., Uchida, K., and Hiratsuka, T. (1976). Cardiac myosin from pig heart ventricle. Purification and enzymatic properties. J. Biochem. (Tokyo) 80, 611-619.

Olson, T. M., Kishimoto, N. Y., Whitby, F. G., and Michels, V. V. (2001). Mutations that alter the surface charge of alpha-tropomyosin are associated with dilated cardiomyopathy. J. Mol. Cell Cardiol. 33, 723-732. doi: 10.1006/jmcc.2000.1339

Orzechowski, M., Fischer, S., Moore, J. R., Lehman, W., and Farman, G. P. (2014). Energy landscapes reveal the myopathic effects of tropomyosin mutations. Arch. Biochem. Biophys. 14, 341-345. doi: 10.1016/j.abb.2014.09.007

Pardee, J. D., and Spudich, J. A. (1982). Purification of muscle actin. Methods Enzymol. 85(Pt B), 164-81. doi: 10.1016/0076-6879(82)85020-9

Perry, S. V. (2001). Vertebrate tropomyosin: distribution, properties and function. J. Musc. Res. Cell Motil. 22, 5-49. doi: 10.1023/A:1010303732441

Pettersen, E. F., Goddard, T. D., Huang, C. C., Couch, G. S., Greenblatt, D. M., Meng, E. C., et al. (2004). UCSF Chimera-a visualization system for exploratory research and analysis. J. Comput. Chem. 25, 1605-1612. doi: $10.1002 /$ jcc. 20084

Phillips, G. N. Jr. (1986). Construction of an atomic model for tropomyosin and implications for interactions with actin. J. Mol. Biol. 192, 128-131. doi: 10.1016/0022-2836(86)90469-9

Phillips, G. N. Jr., Fillers, J. P., and Cohen, C. (1986). Tropomyosin crystal structure and muscle regulation. J. Mol. Biol. 192, 111-131. doi: 10.1016/00222836(86)90468-7

Rajan, S., Ahmed, R. P., Jagatheesan, G., Petrashevskaya, N., Boivin, G. P., Urboniene, D., et al. (2007). Dilated cardiomyopathy mutant tropomyosin mice develop cardiac dysfunction with significantly decreased fractional shortening and myofilament calcium sensitivity. Circ. Res. 101, 205-214. doi: 10.1161/CIRCRESAHA.107.148379 
Redwood, C., and Robinson, P. (2013). Alpha-tropomyosin mutations in inherited cardiomyopathies. J. Musc. Res. Cell Mot. 34, 285-294. doi: 10.1007/s10974-0139358-5

Robinson, P., Griffiths, P. J., Watkins, H., and Redwood, C. S. (2007). Dilated and hypertrophic cardiomyopathy mutations in troponin and alphatropomyosin have opposing effects on the calcium affinity of cardiac thin filaments. Circ. Res. 101, 1266-1273. doi: 10.1161/CIRCRESAHA.107. 156380

Seidman, C. E., and Seidman, J. G. (2011). Identifying sarcomere gene mutations in hypertrophic cardiomyopathy: a personal history. Circ. Res. 108, 743-750. doi: 10.1161/CIRCRESAHA.110.223834

Singh, A., and Hitchcock-Degregori, S. E. (2003). Local destabilization of the tropomyosin coiled coil gives the molecular flexibility required for actin binding. Biochemistry 42, 14114-14121. doi: 10.1021/bi03 48462

Singh, A., and Hitchcock-Degregori, S. E. (2006). Dual requirement for flexibility and specificity for binding of the coiled-coil tropomyosin to its target, actin. Structure 14, 43-50. doi: 10.1016/j.str.2005.09.016

Strzelecka-Golaszewska, H., Jakubiak, M., and Drabikowski, W. (1975). Changes in the state of actin during superprecipitation of actomyosin. Eur. J. Biochem. 55, 221-230. doi: 10.1111/j.1432-1033.1975. tb02154.x

Szczesna, D., Zhang, R., Zhao, J., Jones, M., Guzman, G., and Potter, J. D. (2000). Altered regulation of cardiac muscle contraction by troponin $\mathrm{T}$ mutations that cause familial hypertrophic cardiomyopathy. J. Biol. Chem. 275, 624-630. doi: $10.1074 /$ jbc. 275.1 .624

Tardiff, J. C. (2011). Thin filament mutations: developing an integrative approach to a complex disorder. Circ. Res. 108, 765-782. doi: 10.1161/CIRCRESAHA.110.224170
Urbancikova, M., and Hitchcock-Degregori, S. E. (1994). Requirement of aminoterminal modification for striated muscle alpha-tropomyosin function. J. Biol. Chem. 269, 24310-24315.

Van Driest, S. L., Will, M. L., Atkins, D. L., and Ackerman, M. J. (2002). A novel TPM1 mutation in a family with hypertrophic cardiomyopathy and sudden cardiac death in childhood. Am. J. Cardiol. 90, 1123-1127. doi: 10.1016/S00029149(02)02780-7

Willott, R. H., Gomes, A. V., Chang, A. N., Parvatiyar, M. S., Pinto, J. R., and Potter, J. D. (2010). Mutations in Troponin that cause HCM, DCM AND RCM: what can we learn about thin filament function? J. Mol. Cell Cardiol. 48, 882-892. doi: 10.1016/j.yjmcc.2009.10.031

Conflict of Interest Statement: The authors declare that the research was conducted in the absence of any commercial or financial relationships that could be construed as a potential conflict of interest.

Received: 27 September 2014; accepted: 10 November 2014; published online: 02 December 2014.

Citation: Chang AN, Greenfield NJ, Singh A, Potter JD and Pinto JR (2014) Structural and protein interaction effects of hypertrophic and dilated cardiomyopathic mutations in alpha-tropomyosin. Front. Physiol. 5:460. doi: 10.3389/fphys.2014.00460

This article was submitted to Striated Muscle Physiology, a section of the journal Frontiers in Physiology.

Copyright (c) 2014 Chang, Greenfield, Singh, Potter and Pinto. This is an openaccess article distributed under the terms of the Creative Commons Attribution License (CC BY). The use, distribution or reproduction in other forums is permitted, provided the original author(s) or licensor are credited and that the original publication in this journal is cited, in accordance with accepted academic practice. No use, distribution or reproduction is permitted which does not comply with these terms. 